\title{
Three-Dimensional Stimulated Emission Depletion Microscopy of Nitrogen-Vacancy Centers in Diamond Using Continuous-Wave Light
}

2009

Vol. 9, No. 9

3323-3329

\author{
Kyu Young Han, ${ }^{\dagger, \downarrow}$ Katrin I. Willig, ${ }^{\dagger}$ Eva Rittweger, ${ }^{\dagger}$ Fedor Jelezko, ${ }^{\S}$ \\ Christian Eggeling, ${ }^{*, \dagger}$ and Stefan W. Hell ${ }^{*, \dagger}$ \\ Department of NanoBiophotonics, Max Planck Institute for Biophysical Chemistry, \\ Am Fassberg 11, 37077 Göttingen, Germany, Department of Chemistry, Seoul National \\ University, Seoul 151-747, Korea, and Physikalisches Institut, Universität Stuttgart, \\ 70550 Stuttgart, Germany
}

Received May 20, 2009; Revised Manuscript Received July 16, 2009

\begin{abstract}
Charged nitrogen-vacancy (NV) color centers in diamond are excellent luminescence sources for far-field fluorescence nanoscopy by stimulated emission depletion (STED). Here we show that these photostable color centers can be visualized by STED using simple continuous-wave or high repetition pulsed lasers $(76 \mathrm{MHz}$ ) at wavelengths $>700 \mathrm{~nm}$ for STED. Furthermore, we show that NV centers can be imaged in three dimensions (3D) inside the diamond crystal and present single-photon signatures of single color centers recorded in high density samples, demonstrating a new recording scheme for STED and related far-field nanoscopy approaches. Finally, we exemplify the potential of using nanodiamonds containing NV centers as luminescence tags in STED microscopy. Our results offer new experimental avenues in nanooptics, nanotechnology, and the life sciences.
\end{abstract}

Far-field fluorescence microscopy is one of the most important tools for noninvasive imaging of the interior of transparent objects. However, the resolution of its standard versions is limited by diffraction which precludes discerning similar features that are closer together than about half the wavelength $\lambda$ of the light used, i.e., about $200 \mathrm{~nm}{ }^{1}$ Stimulated emission depletion (STED) microscopy ${ }^{2,3}$ has fundamentally overcome the limits set by diffraction by transiently switching off the fluorescence ability of the dye by stimulated emission, so as to allow neighboring features to be registered sequentially in time by scanning. With standard fluorophores, STED microscopy routinely offers a spatial resolution in the $20-50 \mathrm{~nm}$ range and has successfully been applied to approaching key problems in biology. ${ }^{4,5}$ However, in some applications, STED microscopy is challenged by the limited photostability of the fluorescent markers, which reduces fluorescence brightness and prevents long-term observations. A solution is to employ fluorescent probes with less-photobleaching like luminescent semiconductor quantum dot (QD) nanocrystals ${ }^{6,7}$ or nitrogen-vacancy

* To whom correspondence should be addressed: e-mail, ceggeli@gwdg.de and shell@gwdg.de; phone, +49 551201 2500; fax, +49 5512012505.

${ }^{\dagger}$ Max Planck Institute for Biophysical Chemistry.

* Seoul National University.

$\S$ Universität Stuttgart.
(NV) color centers in diamonds. ${ }^{8}{ }^{8}$ Unfortunately, the fact that the absorption band of most QDs overlaps with that for their emission has hampered their applicability to STED microscopy. In contrast, NV centers in diamond have recently turned out as excellent luminescence sources in STED microscopy, with no observable photobleaching. ${ }^{10}$ Initial STED microscopy images of NV centers in diamond featured an up to $6 \mathrm{~nm}$ spatial resolution in the focal plane $(x, y)$. However, they were recorded inside bulk diamond, with a fixed wavelength of $775 \mathrm{~nm}$ for STED and with a rather low $(8 \mathrm{MHz})$ repetition pulsed laser system. While the low repetition rate may be highly favorable for obtaining the maximum possible resolution with NV centers, it also entails long image acquisition times preventing their application as fluorescent tags in the life sciences and elsewhere. Here, we show that STED nanoscopy on NV centers is viable with tunable continuous-wave (CW) or quasi-CW high-repetitive $76 \mathrm{MHz}$ pulsed laser sources and that it discerns individual $\mathrm{NV}$ centers in diamond crystals with 3D nanoscale resolution. Additionally, we investigate the stimulated emission characteristics of NV centers at different wavelengths and detect the single-photon emission signature of single NV centers inside densely colored samples. The latter introduces a new recording scheme in STED microscopy and related far-field 


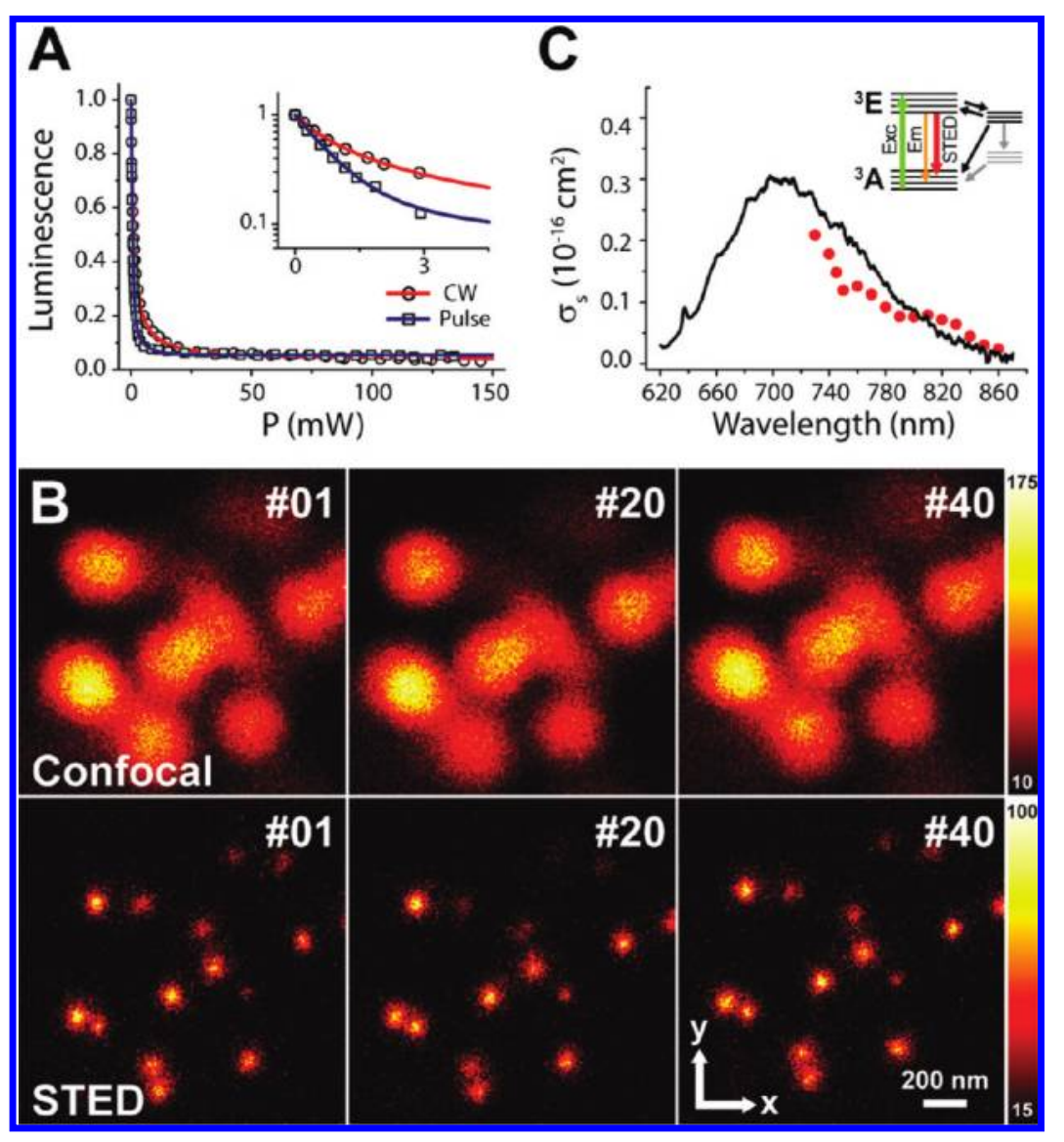

Figure 1. CW STED microscopy of NV centers in bulk diamond. (A) Switch-off (depletion) curve of the luminescence of a single NV center as a function of the time-averaged power $P$ of $\mathrm{CW}$ and $76 \mathrm{MHz}$ pulsed STED light. The drop in luminescence can be described by the function $0.03+0.97 /(1+P / 1.04 \mathrm{~mW})$ for the $\mathrm{CW}$ (red line) and $0.06+0.94 \exp (-P / 0.92 \mathrm{~mW})$ for the pulsed case (blue line), as further depicted in the inset. (B) Consecutive confocal and STED images of densely packed NV centers using CW light $(P=260 \mathrm{~mW}, 150$ $\times 150$ pixels with $10 \mathrm{~nm}$ pixel size and $1 \mathrm{~ms}$ dwell time). The increase in resolution allows separating NV centers in the STED but not in the confocal recordings. Exemplified by the images given for the 20th and 40th scan, the scanning image is the same for over 40 scans, exhibiting the NV centers as extremely photostable. (C) Wavelength-dependence of the cross section of luminescence inhibition calculated from the STED power required to inhibit $50 \%$ of the luminescence (red circles) and the stimulated emission cross section $\sigma_{\mathrm{S}}$ expected from the NVs' luminescence emission spectrum (black line). The good correspondence of both values confirms the stimulated emission nature of the luminescence suppression process. Inset: NV center's energy levels with ground $\left({ }^{3} \mathrm{~A}\right)$ and excited triplet state $\left({ }^{3} \mathrm{E}\right)$, lowest and first excited singlet dark states, vibrational sublevels, and possible interconversion pathways: excitation (Exc), emission (Em), stimulated emission (STED), and dark state transitions.

optical approaches using a targeted switching and read-out modality. ${ }^{11,12}$ Finally, nanoscale images of $\sim 35 \mathrm{~nm}$ sized nanodiamond particles containing NV centers demonstrate the emerging potential of NVs as photostable luminescence tags in far-field fluorescence nanoscopy but also reveal aggregation and heterogeneity of the nanoparticles. Here, the superior resolution of STED microscopy bears itself as a valuable control during optimized nanodiamond production.

The charged NV center (actually $\mathrm{NV}^{-}$) is a unique color center consisting of a vacancy and a nearby nitrogen atom in a diamond network. Excitation of these NV centers at wavelength $\lambda=532 \mathrm{~nm}$ features luminescence with a lifetime $\tau_{\mathrm{f}} \approx 11-12 \mathrm{~ns}$, a high quantum yield $(\approx 0.7)$, a very broad emission spectrum at around $\lambda=700 \mathrm{~nm}$, and exceptional photostability. ${ }^{8,9}$ When excited from its ground $\left({ }^{3} \mathrm{~A}\right)$ to its excited triplet state $\left({ }^{3} \mathrm{E}\right)$, the $\mathrm{NV}$ center relaxes to the vibrational ground state of ${ }^{3} \mathrm{E}$ through efficient phonon coupling (inset Figure 1C). In the presence of the STED light, the luminescence decay $\left(k_{\mathrm{f}}=1 / \tau_{\mathrm{f}}\right)$ competes with the stimulated emission process $\left(k_{\mathrm{S}}=\sigma_{\mathrm{S}} \gamma I\right.$, with photon cross section $\sigma_{\mathrm{S}}$ for stimulated emission and inverse photon energy $\gamma$ of the STED light). If the condition holds that the STED intensity $I$ is much larger than the intensity $I_{\mathrm{S}}=1 /\left(\sigma_{\mathrm{S}} \gamma \tau_{\mathrm{f}}\right)$, stimulated emission dominates over other processes and the ${ }^{3} \mathrm{E}$ is effectively prevented by the STED beam. In the ideal case, the ability of the center to emit spontaneously scales as $\exp \left(-I / I_{\mathrm{S}}\right)$, meaning that the state ${ }^{3} \mathrm{E}$ is prohibited for $I$ $\gg I_{\mathrm{s}}$. As a result, the luminescence ability of the NV center is switched off. To make use of this switch-off process for improving the spatial resolution, the STED light is usually applied with a focal intensity distribution featuring a central zero such as a doughnut. ${ }^{13,14}$ Applying a doughnut beam with intensity maximum $I_{\max } \gg I_{\mathrm{S}}$ reduces the effective spot of luminescence to subdiffraction dimensions, with a full width at half-maximum (fwhm) diameter $d \approx d_{0} /\left(1+C I_{\max } / I_{\mathrm{S}}\right)^{1 / 2}$. Here, $d_{0}$ is the fwhm of a reference spot of a confocal microscope, and $C$ is a constant that depends on the shape of the doughnut minimum. ${ }^{14}$ Consequently, large STED intensities provide high spatial resolution. 
In our previous measurements on the NV centers, we have supplied the STED beam with a fiber laser system operating at $775 \mathrm{~nm}$ and emitting $3.2 \mathrm{~ns}$ pulses at a repetition rate of $8 \mathrm{MHz} .{ }^{10}$ The luminescence inhibition by stimulated emission exhibited the expected steep exponential drop with STED intensity $I$, and the almost unlimited photostability allowed us to apply very large $I$, facilitating a nearly perfect implementation of STED. However, depending on the application, the rather large pulse interdistance of $125 \mathrm{~ns}$, which leads to relatively long image recording times, is disadvantageous. Moreover, selecting a tunable laser source allows for wavelength-dependent measurements and optimization. Hence, we implemented a tunable $\mathrm{CW}$ or quasiCW $76 \mathrm{MHz}$ pulsed laser source and measured the switchoff of a single NV center's luminescence in type IIa chemical vapor deposition (CVD) bulk diamond with increasing power $P$ of the STED light. Excitation of the NV luminescence was supplied by $\mathrm{CW}$ or triggered $76 \mathrm{MHz}(\approx 70 \mathrm{ps})$ pulsed $532 \mathrm{~nm}$ laser light, respectively (Supporting Information).

We found that the steep decrease of the luminescence with the power of the CW or high-repetition STED light is similar to that of the previous low-repetition $8 \mathrm{MHz}$ experiment, again rendering a nearly perfect light mediated switchingoff (Figure 1A). In both the $\mathrm{CW}$ or quasi-CW case, a timeaveraged power $P \approx 0.7-1 \mathrm{~mW}$ and a focal spot area of $\sim 10^{-9} \mathrm{~cm}^{2}$ yield an intensity range of $I_{\mathrm{s}} \approx 0.7-1 \mathrm{MW} / \mathrm{cm}^{2}$ of the STED light which is suitable to switch off half the NV's detected luminescence. In contrast to organic fluorophores requiring $\sim 5$ times higher $\mathrm{CW}$ than $76 \mathrm{MHz}$ quasi$\mathrm{CW}$ powers to accommodate the same NV luminescence inhibition, ${ }^{15}$ with NV centers the power in both modes is nearly the same (1.3-fold larger for $\mathrm{CW})$. One of the reasons for this low factor is the relatively long luminescence lifetime of $\tau_{\mathrm{f}} \approx 11.7 \mathrm{~ns}$ of the NVs (Supporting Information); from the theory a factor of $\left(\sim 76 \mathrm{MHz} \tau_{\mathrm{f}}\right)^{-1}$ is expected. ${ }^{15}$ In the $\mathrm{CW}$ case, the non-negligible constant excitation competing with the inhibition by STED results in a slight deviation from a nearly perfect exponential drop of the luminescence inhibition curve as observed for the $76 \mathrm{MHz}$ repetition STED modality (inset Figure 1A). The dependence of the luminescence inhibition on the CW STED power follows the function $1 /(1+k P)$, as expected from theory (with $k$ denoting a characteristic constant of the luminescent NV). ${ }^{15}$

The level to which we can suppress luminescence is $\sim 3-6 \%$ and slightly larger than with the $8 \mathrm{MHz}$ laser system applied in previous experiments, where complete suppression was observed. The $8 \mathrm{MHz}$ laser system featured $3.2 \mathrm{~ns}$ long pulses, much longer than, for example, the $\sim 300$ ps pulses of the $76 \mathrm{MHz}$ modality. Probably, these long pulses resulted in a more efficient depopulation of all excited states including vibrational substates and additional dark states such as the lowest and first excited singlet states (inset Figure 1C)..$^{9,16-18}$ It is possible that even $\mathrm{CW}$ laser excitation leads to deshelving of the photoexcited singlet state ( $\sim 250 \mathrm{~ns}$ lifetime) into the emitting triplet state. ${ }^{9,16-18} \mathrm{We}$ can, however, exclude two-photon excited NV luminescence or scattered light by the infrared laser light since we have already subtracted such small signal contributions in our data analysis (Supporting Information). Fortunately, the 3-6\% residual level of our present STED modality is low enough not to influence the final STED image. If it were not, one could remove this diffraction-limited contribution to the image by deconvolution or subtraction. ${ }^{19,20}$

The luminescence inhibition curves of Figure 1A represent an average of 15 curves taken on five different single NV centers. While we detected no difference in the steepness of the decays between the different NV centers, a slight variation in the residual fluorescence level became apparent, for example, $1-4 \%$ for the $\mathrm{CW}$ case. Further, the net efficiency of STED also depends on the CW excitation power (Figure S1 in Supporting Information), because in the CW case de-excitation by stimulated emission and excitation are counteracting each other directly. Hence, the STED beam power required to arrange the same luminescence inhibition of the NVs increases with the excitation power. As a result, when the luminescence level of the NV center is increased by increasing the excitation power, slightly larger STED intensities have to be utilized to reach the same resolution. Interestingly, also the residual luminescence level to which suppression is possible increases with the CW excitation power (Figure S1 in Supporting Information); i.e., the higher STED beam intensity cannot fully make up for the stronger excitation. Again, additional dark states such as the singlet states may, besides increasing levels of scattered light by the excitation laser, be involved in this excitation power dependence. Featuring longer lifetimes of, for example, $\sim 100$ ns at room and greater than a microsecond at low temperatures, the dark states are increasingly populated at high excitation power. ${ }^{9,16,17}$ Optically induced depopulation of such dark states ${ }^{16}$ by the STED light may result in fluorescence recovery instead of suppression and counteract the experiment. $^{21}$ Nevertheless, both the $\mathrm{CW}$ and quasi-CW fast repetition rate modalities allow for straightforward recording of super-resolution images.

Figure 1B depicts the scanning images of several NV centers in the bulk diamond. We applied a lateral beam pattern, i.e., a doughnut-shaped intensity distribution of the CW STED light at $740 \mathrm{~nm}$ with a central intensity-zero, and overlaid it with the diffraction-limited spot of the excitation light (Figure S2 in Supporting Information), consequently reducing the focal spot of effective luminescence emission along all lateral directions. ${ }^{14}$ The increased resolution of the STED microscope allowed us to spatially distinguish nearby NV centers that are not resolvable with diffraction-limited confocal recording. At the maximum accessible CW STED power $P=260 \mathrm{~mW}$, these images feature a lateral spatial resolution of $\approx 40 \mathrm{~nm}$. As expected from the resolution scaling with power (Figure S3 in Supporting Information), further downscaling of the resolution may be realized with optimized laser systems, irrespective of CW or pulsed laser, such as with the previously applied high power but lowrepetition pulsed laser system. ${ }^{10}$ Strikingly, again we could continuously record images from the same part of the sample without eliciting any change in the image resolution or luminescence signal due to photobleaching or -blinking, confirming the NV centers as an excellent luminescence 
source for nanoscale STED imaging even with more flexible $\mathrm{CW}$ or high-repetition rate pulsed laser systems. With pixel dwell times of $1 \mathrm{~ms}$, the $1.5 \times 1.5 \mu \mathrm{m}$ images of Figure 1B were recorded within approximately $24 \mathrm{~s}$ and thus $\sim 10$-fold faster than the previous $8 \mathrm{MHz}$ recordings.

The tunable laser source allowed us to investigate further details of the luminescence suppression characteristics of the $\mathrm{NV}$ centers. The wavelength dependence of the suppression cross section of the NV centers' luminescence as experimentally determined by the STED power needed to suppress half of the initial signal follows the values of the stimulated emission cross section $\sigma_{\mathrm{S}}$ expected from the luminescence emission spectrum of the NVs' (Figure 1C, compare Supporting Information), confirming the stimulated emission nature of our luminescence switch-off process. The $>100 \mathrm{~nm}$ difference between excitation and emission of the NV centers' luminescence allows applying wavelengths of the STED light as close as possible to the emission maximum, i.e., with almost the maximum possible efficiency of stimulated emission. However, an increased unspecific background from the bulk diamond rendered applying wavelengths below $740 \mathrm{~nm}$ impractical.

The recording of super-resolution STED images is feasible several micrometers inside the bulk diamond. ${ }^{10}$ In contrast to our previous STED recordings with purely lateral confinement, the application of an axial beam pattern, i.e., a focal intensity distribution of the STED light confining the focal spot of effective luminescence emission strongly along the $z$-direction, allows for an additional resolution enhancement (Figure S2 in Supporting Information). ${ }^{3,14}$ Implementing this STED beam pattern, super-resolution images of NV centers $>6 \mu \mathrm{m}$ deep inside the bulk diamond show a clear separation of single centers in 3D (Figure 2). Lateral and axial extension of the resolved spots is about 110-120 nm and 110-135 $\mathrm{nm}$ (depending on the depth), respectively, thus revealing an almost isotropic effective focal spot with a $\sim 2$ - and 5-6fold improvement over conventional confocal recordings, respectively. ${ }^{3}$ The 3D images were recorded with highrepetition pulsed excitation and STED light. Similarly, highrepetition pulsed excitation can be combined with CW STED light (Figure S4 in Supporting Information). The straightforward applicability of pulsed luminescence excitation indicates the possibility of combining time-resolved luminescence methods with nanoscale resolution..$^{22}$

$\mathrm{NV}$ color centers are reliable single photon sources at room temperature and in recent years they have been widely used in quantum cryptography and computation. ${ }^{9,17,23}$ Maintaining high resolution in the far-field, such experiments can also be performed with STED microscopy. We recorded the photon correlation from single isolated spots of our images of the NV centers in the bulk diamond using a Hanbury Brown-Twiss detection setup (Supporting Information). ${ }^{24,25}$ With pulsed excitation, a peak at zero delay time of the crosscorrelation function $G^{(2)}(\tau)$ of the recorded luminescence signal is absent only for a single emitter. ${ }^{23,26,27}$ This strong antibunching is a clear indication of the fact that, due to its finite excited state lifetime, a single emitter such as a single isolated NV center cannot emit two photons at the same time.

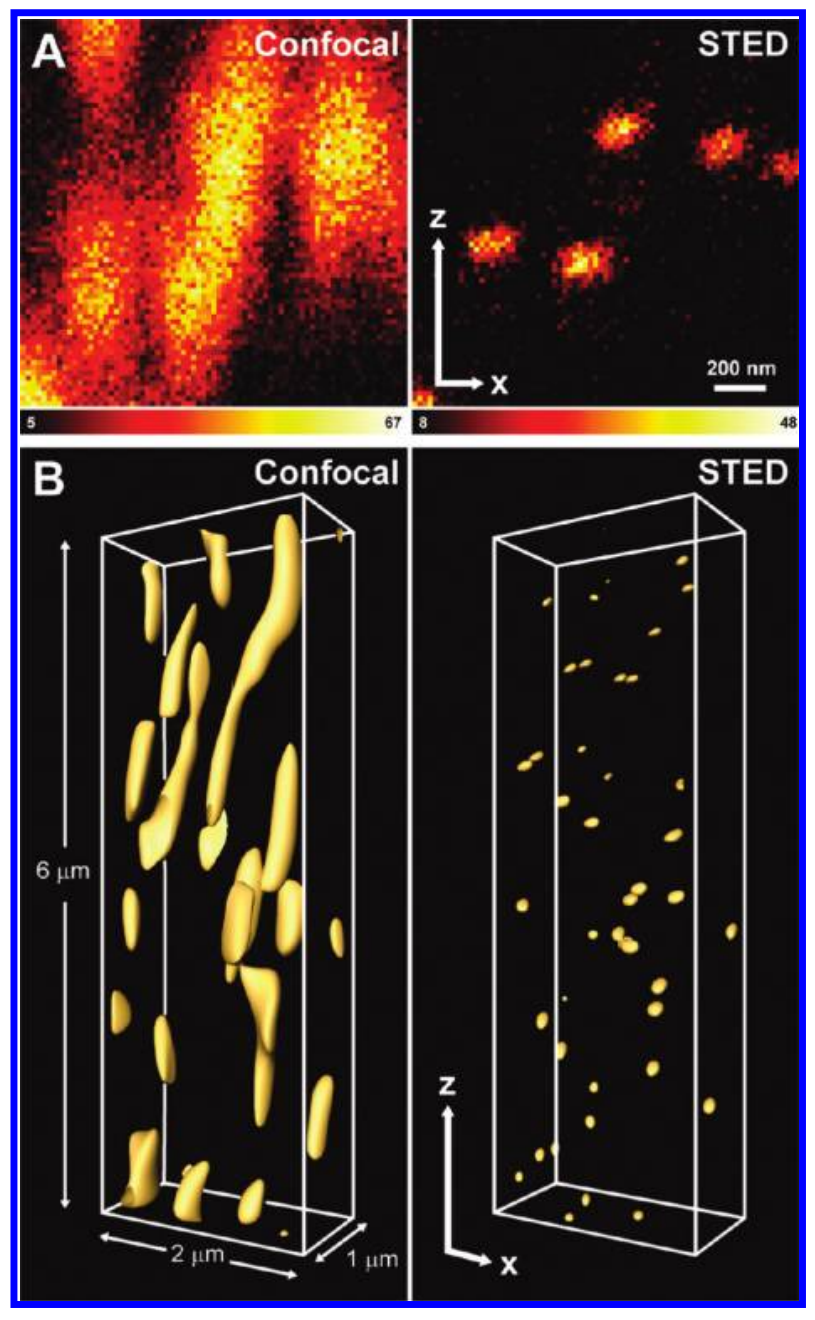

Figure 2. 3D STED microscopy of NV centers in bulk diamond. (A) Individual color centers are sharply resolved in the $x z$-profile of a STED image (right) and blurred in the confocal recording (left) ( $P=100 \mathrm{~mW}, 10 \mathrm{~nm}$ pixel size and $0.5 \mathrm{~ms}$ dwell time). (B) 3D surface-rendered representation of a bulk of NV centers by confocal (left) and STED (right) microscopy.

A single photon signature may in principle also be observed for multichromophoric systems via efficient annihilation processes between chromophores in close proximity. ${ }^{28} \mathrm{We}$ can exclude this possibility in our CVD bulk diamond sample, where the probability for very close lying color centers is low.

In both confocal and STED recordings, we could not detect a notable peak at $G^{(2)}(0)$ from a single isolated NV (Figure S5 in Supporting Information). However, in a more densely NV-labeled sample, the peak is only absent in the STED recording, whose resolution power is strong enough to separate neighboring NVs (Figure 3A), in contrast to that of the confocal recording. We have used a repetition rate of 10.9 $\mathrm{MHz}$ in our excitation to clearly separate neighboring peaks in $G^{(2)}(\tau)$. Our cross-correlation data $G^{(2)}(\tau)$ can be well described by eq 1

$$
G^{(2)}(\tau)=A+B \sum_{j=-1}^{1} \exp \left[-|\tau-j(1 / f)| / \tau_{\mathrm{f}}\right]-D \exp \left[-\tau / \tau_{\mathrm{f}}\right]
$$




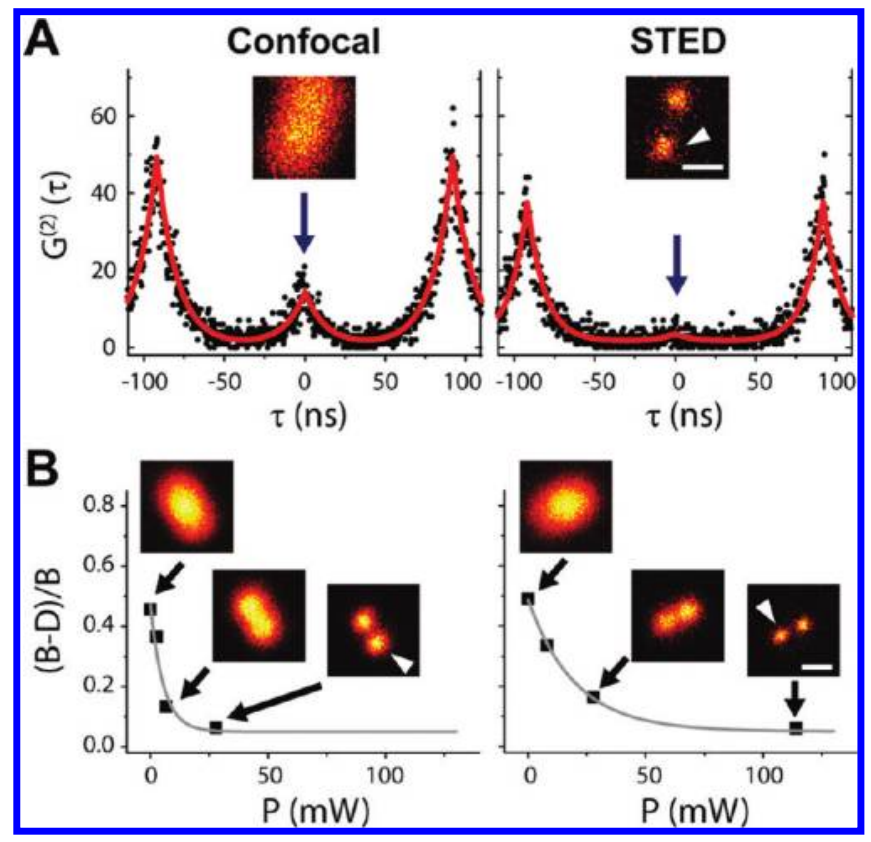

Figure 3. A tunable focal spot of STED microscopy allows distinction of multiple single emitters in dense NV samples. Correlation function $G^{(2)}(\tau)$ of the luminescence signal recorded with a Hanbury Brown-Twiss detection setup. (A) Photon correlation of NV centers in bulk diamond with confocal (left) and STED (right) detection: Only STED can clearly separate two $\sim 240 \mathrm{~nm}$ spatially separated NVs, demonstrated by the negligible peak at zero lag time $G^{(2)}(0)$ and further revealed by fits (red line) of eq 1 to the data (see text). (B) New recording modality for STED or related far-field nanoscopy approaches: for a clear separation of two neighboring objects, the switch-off (STED) light power $P$ has only to be increased until the relative peak height $(B-D) / B$ at $G^{(2)}(0)$ reaches a certain low value, as exemplified for two $165 \mathrm{~nm}$ (left panel) and $140 \mathrm{~nm} \mathrm{NV}$ centers (right panel). Insets: Respective images of the NV centers (scale bar $=200 \mathrm{~nm}$ ); the arrows mark the focal position (white arrows) or the STED power $P$ (black arrows) during the recordings.

with offset $A$, height $B$ of the peaks at nonzero lag times $\tau$, relative reduction $D$ of the peak height at $\tau=0$, and repetition rate $f$ of the excitation. ${ }^{27}$ This fit gives a good estimate of (i) the luminescence lifetime $\tau_{\mathrm{f}}$ of the NV center, which correlates with the width of the peaks and whose resulting values of $10-12$ ns coincide well with $\tau_{\mathrm{f}}=11.7$ ns determined by a time-correlated single photon counting (TCSPC) (Supporting Information), and (ii) the relative peak height at zero lag time $(B-D) / B$, which is determined to be 0.3 in the confocal recording of Figure $3 \mathrm{~A}$ and $0.04-0.07$ in the other recordings. Values of $(B-D) / B<0.1$ indicate a single photon emitter, and the nonzero value results from background photons and the finite laser pulse width. ${ }^{26}$ On the contrary, a value $(B-D) / B=0.3$ approximately reveals 1.4 independent $\mathrm{NV}$ emitters, ${ }^{26}$ which is to be expected from the confocal overlapping image of two $\sim 240 \mathrm{~nm}$ distant NV centers (insets Figure 3A). The ability to perform these simple quantum optical experiments in rather high density samples exemplifies the power of combining NV luminescence and STED microscopy. Further, it allows us to indicate a new image recording scheme for separating single luminescent sources. For each scanning pixel, we only need to apply a STED intensity $I_{\max }$ that ensures a level where $G^{(2)}(0)$ appears to be zero, as outlined for two $165 \mathrm{~nm}$ or $140 \mathrm{~nm}$ separated NV centers (Figure 3B). In the $140 \mathrm{~nm}$ case approximately $P=65 \mathrm{~mW}$ of STED light is necessary to clearly separate the two centers, while in the $165 \mathrm{~nm}$ case much less $P=20 \mathrm{~mW}$ is sufficient $(B-D) / B \approx 0.05)$. For an accurate localization of the neighboring spots even less STED power may be acceptable, which can, for example, be adjusted following slightly larger values of $(B-D) / B \approx$ 0.2 . This procedure thus allows for an optimized adaptation of the STED light in the scanning image acquisition process, potentially avoiding unnecessarily high intensity values.

STED microscopy on NV centers not only may help in elucidating solid-state systems but also may be applied to the life sciences. Nanodiamond particles with single or multiple NV centers and sizes down to a few nanometers may potentially be employed as biomarkers in living cells. ${ }^{29-32}$ However, characteristics such as aggregation or heterogeneity in luminescence properties are currently impeding the use of nanodiamonds as luminescent markers. We have prepared samples of $\sim 35 \mathrm{~nm}$ sized nanodiamonds with multiple NV centers ${ }^{32}$ in poly(vinyl-alcohol) (PVA) (Supporting Information). Following $532 \mathrm{~nm} \mathrm{CW}$ excitation, inhibition of luminescence of these nanodiamonds by $\mathrm{CW}$ STED light at $740 \mathrm{~nm}$ is as efficient as for the NV centers in bulk diamond (Figure 4A). Due to, for example, the increased luminescence lifetime of the NV-nanodiamonds having a major component of $\sim 18 \mathrm{~ns}$ (Supporting Information), the STED power $P \approx 0.8 \mathrm{~mW}$ needed to suppress half of the nanodiamonds' luminescence is $\sim 1.3$ times lower than that of the NV centers in bulk diamond (inset Figure 4A). The depletion curve by the STED light (Figure 4A) is an average of 24 curves taken from eight different single nanodiamonds. All individual curves differ slightly from each other. This inhomogeneity is also confirmed by slight variations in the luminescence decay from one nanodiamond to the other; all decays can anyway only be described by at least two components (Supporting Information). Therefore, the averaged depletion curve shows slight deviation from the theoretically expected function $1 /(1+k P$ ) (Figure 4A).

STED images of the nanodiamonds show significant enhancement in resolution over confocal recordings (Figure 4B). The isolation of single spots in our STED images depicts an improved ability of recognizing heterogeneity in spot size and brightness of the nanodiamond particles. The diversity of the imaged particles renders itself in a much wider distribution of values of their luminescence lifetime and particle size as compared to the values depicted from single NV centers in bulk diamond (Figure 4C). An increase in particle size suggests aggregation of several particles. The accompanying decrease of the average luminescence lifetime may result from the closer proximity of several NV centers or due to surface effects. The pronounced variation in luminescence lifetime values (even for the same particle size) as well as the noncorrelative characteristic of lifetime or particle size with luminescence brightness (Figure S6 in Supporting Information) demonstrate further differences in the composition of each nanodiamond with respect to the number of NV centers and their charges or distances to the 


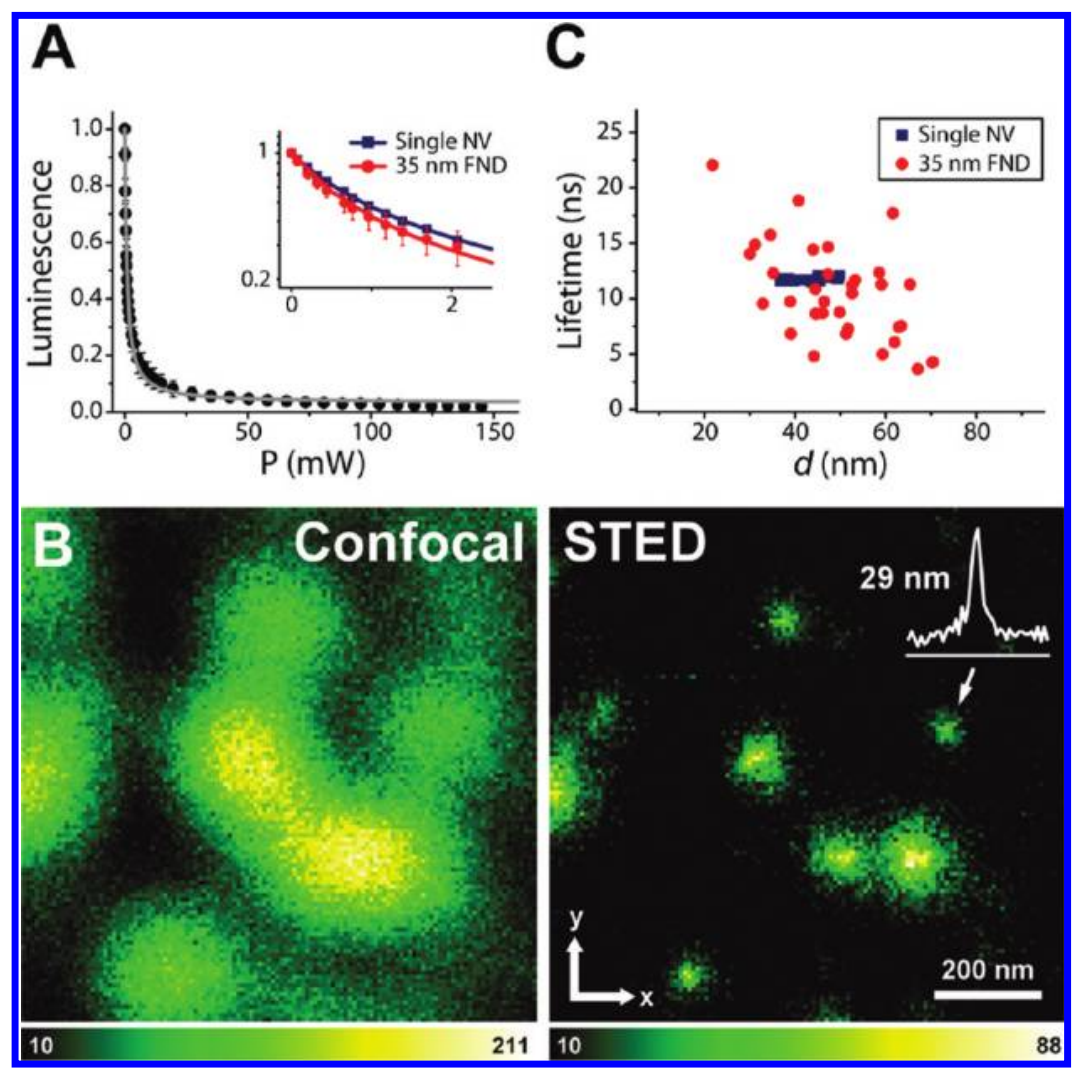

Figure 4. STED microscopy on $\sim 35 \mathrm{~nm}$ sized luminescent nanodiamonds in PVA. (A) Excited-state luminescence inhibition of the nanodiamonds as a function of the power $P$ of CW STED light. The curve is an average of 24 curves recorded on eight individual nanodiamonds. The error bars representing the standard deviation between the individual curves indicate slight inhomogeneities, which result in a slightly imperfect description of the drop in luminescence by the function $0.03+0.97 /(1+P / 0.82 \mathrm{~mW})($ gray line). Inset: Compared to the single NV centers in bulk diamond, the luminescence suppression curve of the nanodiamonds ( $35 \mathrm{~nm}$ FND) is shifted to a slightly lower STED power. (B) CW STED images of the nanodiamonds, clearly resolved in the STED (right) but blurred in the confocal recording (left) $(P=260 \mathrm{~mW}, 150 \times 150$ pixels of $10 \mathrm{~nm}$ pixel size and $0.5 \mathrm{~ms}$ dwell time $)$. Some spots reveal single nanodiamonds as exemplified by the $x$-line profile over one of the spots, while larger and brighter spots may result from several aggregated nanodiamond particles or other differences in the particles composition. (C) Heterogeneities of the nanodiamonds are further outlined by the correlative plot of value pairs of average luminescence lifetime and particle size $d$ determined from 34 spots selected from the STED images of the $35 \mathrm{~nm}$ nanodiamonds (red circles) and from 16 spots selected from the STED images of the NV centers in bulk diamond (blue squares); $19 \mathrm{MHz}$ pulsed excitation and $76 \mathrm{MHz}$ STED light at $P=160 \mathrm{~mW}$, correlation coefficient of -0.576 for nanodiamonds. Variations of the spot size $d$ of the NV centers in bulk diamond result from differences of the positions of the centers with respect to the focal plane.

particle surface, or due to surface inhomogeneities and contaminations. ${ }^{32}$ As for the bulk diamond, subsequent recordings reveal no sign of photobleaching of the luminescent nanodiamond particles. The so far unreached possibility of STED microscopy to monitor inhomogeneity of optical properties and nanoparticle size will improve control on elaborate nanodiamond production, and subsequent pertinent surface functionalization ${ }^{31-34}$ or size-downscaling ${ }^{35}$ may enable specific biomolecular labeling.

In summary, we have shown that the photophysical characteristics of the NV centers allow their efficient use for STED microscopy with tunable CW or quasi-CW highrepetitive pulsed laser sources. Under these experimental conditions, the NV centers exhibit no sign of photobleaching allowing for a multitude of scans of the same part without compromising signal or spatial resolution. As a consequence, 3D super-resolution images of single NV centers are directly accessible, and the clear single-photon emission signature of a single NV center can now also be recorded in rather dense samples, allowing for an optimized scanning image recording scheme. In addition,
STED microscopy of nanodiamond particles with single or multiple NV centers demonstrates that they can potentially be utilized as specific markers in life science experiments. The relatively long luminescence lifetime of the NVs offers easy access to multilabel and consequently colocalization imaging, since time gating may distinguish signals from various labels based on their distinct luminescence lifetimes. ${ }^{32}$

Nanoscopy on single NV centers not only includes pure imaging but also enables spectroscopic and dynamical studies such as fluorescence correlation spectroscopy on the nanoscale. ${ }^{5,36}$ Besides stimulated emission depletion (or ground state depletion ${ }^{37}$ ), other candidate mechanisms that could facilitate nanoscopy by NV luminescence switching ${ }^{12,38}$ are their photochromic transitions (to neutral NV centers) ${ }^{39}$ spin orientation properties, ${ }^{40,41}$ or any other transient shelving into one of their dark states. ${ }^{42,43}$ Other defect centers in diamond may also lend themselves to overcoming the resolution limits set by diffraction. The viability of photobleaching-free farfield fluorescence 3D nanoscopy with simple laser sources should provide a whole new range of opportunities to explore 
the nanoscale in the material and the life sciences in three dimensions.

Acknowledgment. We thank Jay Jethwa, Gael Moneron, Ben Harke, and Scott Irvine for help with the experiments, Andreas Schönle for support with the Imspector software, and Daniel Twitchen (Element 6) for providing a polycrystalline diamond sample. K.Y.H. gratefully acknowledges support from the Korea Research Foundation Grant funded by the Korean Government (MOEHRD).

Supporting Information Available: Details on the sample preparation, optical setup, measurement performance and determination of the luminescence lifetime and the cross sections of stimulated emission and Figures S1-S6 depicting the dependence of the luminescence switch-off of the NV's luminescence by CW STED light on the excitation power (Figure S1), the focal intensity profiles of the STED light (Figure S2), the resolution scaling of NV imaging with STED power (Figure S3), the luminescence switch-off by the STED light for a single NV center in the bulk diamond applying pulsed excitation and CW STED light (Figure S4), antibunching experiments on single isolated NV center (Figure S5), and correlative plots of luminescence lifetime or particle size versus luminescence brightness for $35 \mathrm{~nm}$ nanodiamonds in PVA and NV centers in bulk diamond (Figure S6). This material is available free of charge via the Internet at http:// pubs.acs.org.

\section{References}

(1) Abbe, E. Arch. Mikrosk. Anat. 1873, 9, 413-468.

(2) Hell, S. W.; Wichmann, J. Opt. Lett. 1994, 19 (11), 780-782.

(3) Klar, T. A.; Jakobs, S.; Dyba, M.; Egner, A.; Hell, S. W. Proc. Natl. Acad. Sci. U.S.A. 2000, 97, 8206-8210.

(4) Willig, K. I.; Rizzoli, S. O.; Westphal, V.; Jahn, R.; Hell, S. W. Nature 2006, 440 (7086), 935-939.

(5) Eggeling, C.; Ringemann, C.; Medda, R.; Schwarzmann, G.; Sandhoff, K.; Polyakova, S.; Belov, V. N.; Hein, B.; von Middendorff, C.; Schonle, A.; Hell, S. W. Nature 2009, 457, 1159-1163.

(6) Chan, W. C. W.; Nie, S. M. Science 1998, 281, 2016-2018.

(7) Michalet, X.; Pinaud, F. F.; Bentolila, L. A.; Tsay, J. M.; Doose, S.; Li, J. J.; Sundaresan, G.; Wu, A. M.; Gambhir, S. S.; Weiss, S. Science 2005, 307 (5709), 538-544.

(8) Gruber, A.; Drabenstedt, A.; Tietz, C.; Fleury, L.; Wrachtrup, J.; von Borczyskowski, C. Science 1997, 276, 2012-2014.

(9) Jelezko, F.; Wrachtrup, J. Phys. Status Solidi A 2006, 203, 32073225.

(10) Rittweger, E.; Han, K. Y.; Irvine, S. E.; Eggeling, C.; Hell, S. W. Nat. Photonics 2009, 3, 144-147.

(11) Hell, S. W.; Dyba, M.; Jakobs, S. Curr. Opin. Cell. Biol. 2004, 14 (5), 599-609.

(12) Hell, S. W. Science 2007, 316 (5828), 1153-1158.

(13) Hell, S. W. Nat. Biotechnol. 2003, 21 (11), 1347-1355.

(14) Harke, B.; Keller, J.; Ullal, C. K.; Westphal, V.; Schoenle, A.; Hell, S. W. Opt. Express 2008, 16 (6), 4154-4162.

(15) Willig, K. I.; Harke, B.; Medda, R.; Hell, S. W. Nat. Methods 2007, 4 (11), 915-918.
(16) Drabenstedt, A.; Fleury, L.; Tietz, C.; Jelezko, F.; Kilin, S.; Nizovtzev, A.; Wrachtrup, J. Phvs. Rev. B 1999, 60 (16), 11503-11508.

(17) Kurtsiefer, C.; Mayer, S.; Zarda, P.; Weinfurter, H. Phvs. Rev. Lett. 2000, 85 (2), 290-293.

(18) Rogers, L. J.; Armstrong, S.; Sellars, M. J.; Manson, N. B. New J. Phvs. 2008, 10, 103024.

(19) Hell, S. W., Increasing the resolution of far-field fluorescence light microscopy by point-spread-function engineering. In Topics in Fluorescence Spectroscopy, Lakowicz, J. R., Ed.; Plenum Press: New York, 1997; Vol. 5, pp 361-422.

(20) Hofmann, M.; Eggeling, C.; Jakobs, S.; Hell, S. W. Proc. Natl. Acad. Sci. U.S.A. 2005, 102 (49), 17565-17569.

(21) Ringemann, C.; Schonle, A.; Giske, A.; von Middendorff, C.; Hell, S. W.; Eggeling, C. ChemPhvsChem 2008, 9 (9), 612-624.

(22) Auksorius, E.; Boruah, B. R.; Dunsby, C.; Lanigan, P. M. P.; Kennedy, G.; Neil, M. A. A.; French, P. M. W. Opt. Lett. 2008, 33 (2), 113115.

(23) Beveratos, A.; Brouri, R.; Gacoin, T.; Villing, A.; Poizat, J. P.; Grangier, P. Phys. Rev. Lett. 2002, 89, 187901.

(24) Hanbury Brown, R.; Twiss, R. Q. Nature 1956, 177 (4497), 27-29.

(25) Hell, S. W.; Soukka, J.; Haenninen, P. E. Bioimaging 1995, 3, 6569.

(26) Weston, K. D.; Dyck, M.; Tinnefeld, P.; Muller, C.; Herten, D. P.; Sauer, M. Anal. Chem. 2002, 74 (20), 5342-5349.

(27) Sykora, J.; Kaiser, K.; Gregor, I.; Bonigk, W.; Schmalzing, G.; Enderlein, J. Anal. Chem. 2007, 79 (11), 4040-4049.

(28) Masuo, S.; Vosch, T.; Cotlet, M.; Tinnefeld, P.; Habuchi, S.; Bell, T. D. M.; Oesterling, I.; Beljonne, D.; Champagne, B.; Muellen, K.; Sauer, M.; Hofkens, J.; De Schryver, F. C. J. Phvs. Chem. B 2004, 108 (43), 16686-16696.

(29) Beveratos, A.; Brouri, R.; Gacoin, T.; Poizat, J. P.; Grangier, P. Phvs. Rev. A: At., Mol., Opt. Phvs. 2001, 64, 061802.

(30) Kuehn, S.; Hettich, C.; Schmitt, C.; Poizat, J. P.; Sandoghdar, V. J. Microsc. (Oxford, U.K.) 2001, 202 (1), 2-6.

(31) Neugart, F.; Zappe, A.; Jelezko, F.; Tietz, C.; Boudou, J. P.; Krueger, A.; Wrachtrup, J. Nano Lett. 2007, 7 (12), 3588-3591.

(32) Fu, C. C.; Lee, H. Y.; Chen, K.; Lim, T. S.; Wu, H. Y.; Lin, P. K.; Wei, P. K.; Tsao, P. H.; Chang, H. C.; Fann, W. Proc. Natl. Acad. Sci. U.S.A. 2007, 104 (3), 727-732.

(33) Krueger, A. Chem. Eur. J. 2008, 14, 1382-1390.

(34) Vial, S.; Mansuy, C.; Sagan, S.; Irinopoulou, T.; Burlina, F.; Boudou, J. P.; Chassaing, G.; Lavielle, S. ChemBioChem 2008, 9, 2113-2119.

(35) Smith, B. R.; Inglis, D. W.; Sandnes, B.; Rabeau, J. R.; Zvyagin, A. V.; Gruber, D.; Noble, C. J.; Vogel, R.; Osawa, E.; Plakhotnik, T. Small 2009, DOI: $10.1002 / \mathrm{smll} .200801802$.

(36) Kastrup, L.; Blom, H.; Eggeling, C.; Hell, S. W. Phvs. Rev. Lett. 2005, 94, 178104.

(37) Rittweger, E.; Wildanger, D.; Hell, S. W. EPL 2009, 86, 14001.

(38) Hell, S. W. Nat. Methods 2009, 6 (1), 24-32.

(39) Gaebel, T.; Domhan, M.; Wittmann, C.; Popa, I.; Jelezko, F.; Rabeau, J.; Greentree, A.; Prawer, S.; Trajkov, E.; Hemmer, P. R.; Wrachtrup, J. Appl. Phvs. B: Lasers Opt. 2006, 82 (2), 243-246.

(40) Taylor, J. M.; Cappellaro, P.; Childress, L.; Jiang, L.; Budker, D.; Hemmer, P. R.; Yacoby, A.; Walsworth, R.; Lukin, M. D. Nat. Phvs. 2008, 4, 810-816.

(41) Balasubramanian, G.; Chan, I. Y.; Kolesov, R.; Al-Hmoud, M.; Tisler, J.; Shin, C.; Kim, C.; Wojcik, A.; Hemmer, P. R.; Krueger, A.; Hanke, T.; Leitenstorfer, A.; Bratschitsch, R.; Jelezko, F.; Wrachtrup, J. Nature 2008, 455, 648-651.

(42) Hell, S. W.; Kroug, M. Appl. Phvs. B: Lasers Opt. 1995, 60, 495497.

(43) Bretschneider, S.; Eggeling, C.; Hell, S. W. Phvs. Rev. Lett. 2007, 98, 218103.

NL901597V 